\title{
Thermal Energy Harvesting - Infinite Power for ubiquitous Monitoring Sensors
}

\author{
Dr. Nurnus, Joachim / Habbe, Burkhard \\ Micropelt GmbH \\ Emmy-Noether-Str. 2 \\ 79110 Freiburg, Germany
}

Wireless sensor systems continue to increase their market share in applications ranging from smart metering and building automation to industrial condition and process monitoring. However, actual market growth continuously lags behind most forecasts. Energy constraints caused by batteries rank amongst the main concerns issued by potential users. Is energy harvesting a solution to those concerns and can this young and expanding technology serve as an enabler to ubiquitous sensing?

Micropelt, a Freiburg, Germany based specialist in thin-film thermoelectrics specializes in harvesting energy from excess heat which could be generated by hot processes, friction of all kinds, energy production and transport, combustion, resistive heat and many more sources. The fundamental element of Micropelt's technology is their patented combination of advanced thermoelectric material science and wafer processing technologies.

A patented process sequence deposits a thin film of thermoelectric Bismuth-Telluride $\left(\mathrm{Be}_{2} \mathrm{Te}_{3}\right)$ which then undergoes a patented microstructuring, MEMS-like process. Wafer parts are bonded together and eventually yield chip-sized devices with economies-of-scale similar to

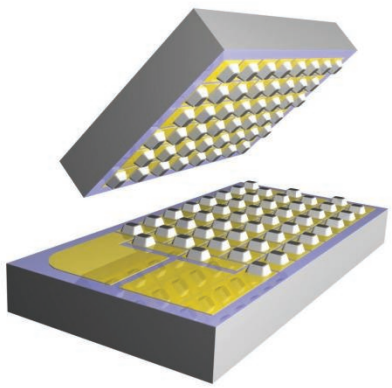
conventional microelectronics. . With feature sizes as small as $35 \mu \mathrm{m}$ Micropelt's technology creates the world's highest density of up to 50 thermocouples per square millimeter. Consequently the voltage per sqmm is 400 to 600 times higher than that of conventional thermoelectric devices. The active footprint of Micropelt's MPG-D751

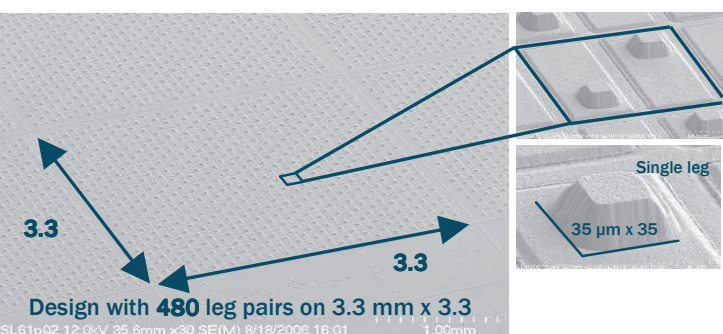
thermogenerator is less than $11 \mathrm{~mm}^{2}$ and still contains 540 thermocouples. 10 Kelvin $(K)$ of temperature differential applied to this device produces 1.4 Volt open circuit voltage and about $2 \mathrm{~mW}$ of power at matched loads of 300 Ohms. Small form factor and high voltage density support compact mechanical and electronic integration much better than conventional TE devices.

As a blueprint for a fully autonomous self-sustained wireless sensor node Micropelt has designed the modular wireless thermoharvesting evaluation system TE-Power NODE. It has been conceived to facilitate first steps of both users and development engineers into application as well as design related aspects of thermal energy harvesting. Consequently the TE-Power Node system got a high degree of flexibility through a still increasing number of power conditioning modules, wireless sensor modules and a custom application interface module. All these pluggable components support the evaluation of thermoharvesting in both lab and field, along with the exploration of power budgets, the development of thermoharvesting driven integrated and distributed systems, or the simulation of harvester-compliant duty cycles for new or already installed transmitters. Custom wireless applications can be matched by fixed or adjustable supply voltages, expandable storage capacitance and the respective available power budget.

To operate the wireless sensor node, it must be attached to a suitable heat source which offers a so-called minimum gross gradient of 8 to $10^{\circ} \mathrm{C}$ between the warm surface and ambient air. After a few seconds the TE Power Node begins transmitting information to a PC via a standard USB dongle receiver. With its minimized protocol stack it only takes 2 milliseconds to measure and transmit the temperature of the thermogenerator's hot and cold sides once every second along with the respective buffer capacitor voltage. Thermal power consumption, in other words heat flux, and electrical output data such as voltage and power are displayed by the PC based user interface software TEPower SCOPE which converts the cyclical data stream from up to five linked devices into each individual diagrams. Direct logging to disk allows the user to properly assess the particular harvesting location's thermal behavior over virtually unlimited periods of time. Additional digital sensors can be attached to the TE-Power NODE's wireless module via its $\mathrm{I}^{2} \mathrm{C}$ bus interface. Transmission of data such as vibration, humidity or pressure 
data, as the respective sensors permit, can be performed. The extra power needed is well within the range of the harvester. A practical example was just released by Micropelt. The TE-Ppower NODE VM demonstrates the concept of wireless and battery-free condition monitoring, powered by heat harvested from the monitored machine. Although its sampling frequency of $400 \mathrm{~Hz}$ limits the scope of applications it still proves the concept by clearly distinguishing a good vacuum pump from a damaged one.
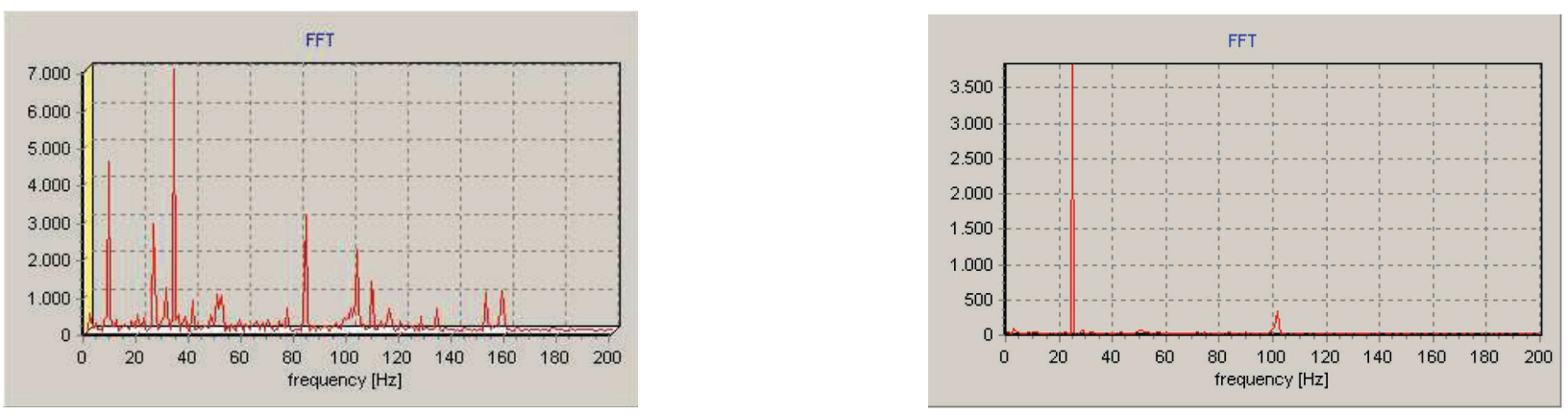

Optimal locations for measurements and energy harvesting are often not identical. Hence, a distributed architecture of a harvester-based system is an obvious need. Micropelt provides a thermoharvesting power supply, TE-Power PROBE, which delivers energy to the powered instrument through a short wire. This external harvester was designed to drive wireless systems in replacement of a primary battery pack. Thus the harvester can be mounted where sufficient waste energy is readily available without limiting the optimal positioning of a sensor. At its far end the cable connects to an adapter device which presents battery-like power to the client instrument and simply slips into the

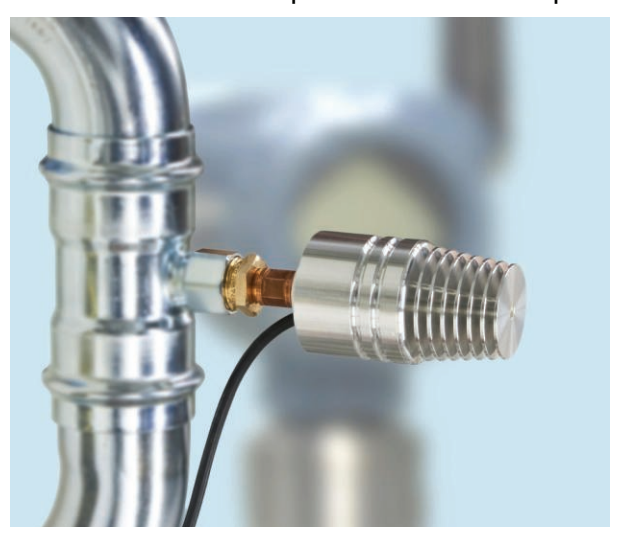
original battery slot - unless the instrument features a generic energy harvesting input anyway. Both, the adapter and the generic interface are currently subject to standardization efforts under Micropelt's participation.

$\mathrm{ABB}$, one of the leading manufacturers of control instrumentation, concentrated on the integrated aspect of energy harvesting powered devices. As a result of their collaboration with Micropelt a fully integrated thermoharvesting wireless temperature transmitter was presented as a technology demonstrator - receiving an industry award right after its introduction. The unit integrates Micropelt's TEGs, the thermal path and a heat sink into its usual process connection and thus internally generates the power used by its also built-in thermopile and WirelessHART transmitter. Mounting and commissioning this instrument is reduced to just screwing it into its receptacle at a predefined measurement position, where a temperature differential of approximately $30^{\circ} \mathrm{C}$ is available for self-sustaining operation.

Thermoharvesting based on high voltage thermogenerators is on its way to main stream. Evaluation prior to use is made easy by modular evaluation systems, keeping risks low. Micropelt are about to open their first volume production line for millions of thin film thermoelectric devices, so

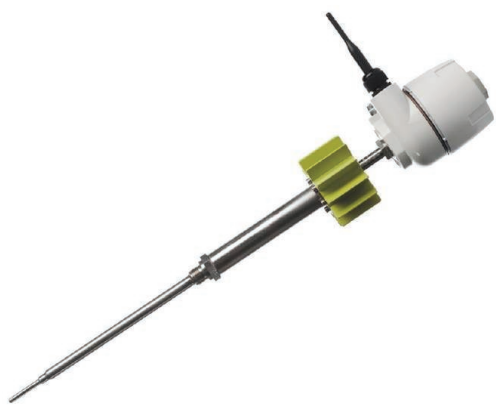
thermoharvesting volume products can be served. With growing understanding and acceptance of duty cycle based process and condition monitoring sensors and their enormous upside in operations, maintenance and overall efficiency improvement the market is poised for the previously forecasted growth rates. Maybe wireless sensors will be for equipment tomorrow what mobile phones are for humans today. 